\title{
3D CAUCHY PROBLEM FOR AN ELASTIC LAYER: INTERFACIAL CRACKS DETECTION
}

\author{
ALEXANDER N. GALYBIN ${ }^{1,2} \&$ SERGEY M. AIZIKOVICH ${ }^{2}$ \\ ${ }^{1}$ The Schmidt Institute of Physics of the Earth (IPE RAS), Moscow, Russia \\ ${ }^{2}$ Don State Technical University, Russian Federation
}

\begin{abstract}
This study presents a Cauchy-type problem of 3D elasticity for an elastic layer that can be bonded to an infinite base (half-space) made of dissimilar elastic material. The initial conditions are given on one side of the layer and both stress and displacement vectors are assumed to be known simultaneously. No conditions are specified on the other side. In the case of this side being fully bonded to the base, the stress and displacement vectors are continuous across the interface. This fact introduces certain relationships that have to be imposed on the initial conditions in order to obey continuity. We use these in order to detect a possible appearance of delamination of the interface. By using the double Fourier transform and the general solution of 3D elasticity in terms of harmonic functions, the initial value problem is reduced to a system of Fredholm integral equations of the first kind. Solutions of such systems are usually unstable; therefore, a numerical approach is suggested to overcome this difficulty by using the SVD regularisation. A possibility of delamination detection is discussed.
\end{abstract}

Keywords: layered elastic structures, interfacial cracks, inverse problems, integral equations, regularisation.

\section{INTRODUCTION}

This study continues the previous research for a 2D elastic strip [1] and generalises it for the $3 \mathrm{D}$ case of an elastic layer that can also be bonded to an elastic base (half-space) made of a different material. The main focus of this study is the development of mathematical methods capable of crack (delamination) detection on the interface between the layer and the base by using the strain/displacement measurements on the stress-free surface of the layer. Such a problem belongs to a wide class of incorrectly posed inverse problems of elasticity. It is known that the problem with overdetermined boundary conditions on a part of the boundary is incorrectly posed and can be unsolvable or possess unstable solutions. There are many publications regarding 2D inverse problems of plane elasticity but not so many for the 3D case. Standard formulation of the Cauchy initial value problem of plane elasticity usually assumes that the displacements vector $\mathbf{u}$ is known together with the stress vector $\mathbf{p}$ on a part of the boundary (usually it is stress-free, i.e. $\mathbf{p}=0$ ) and no boundary conditions are specified on the rest of the boundary. Shvab [2] referred to such mathematical formulations as the $(\mathbf{u}, \mathbf{p})$ problem. Some other formulations, (see survey in Bonnet and Constantinescu [3]), can be reduced to the (u,p) problem. It is evident that in the case of plane elasticity the $(\mathbf{u}, \mathbf{p})$ problem can be routinely solved by a finite difference method applied to the Cauchy type system of two first-order differential equations of equilibrium, three linear Hook's law equations and the three first order strain-displacement equations. In the case of $3 \mathrm{D}$, the number of equations in the system increases but this would not present essential difference for the numerical analysis. However, such finite difference approaches require essential modifications due to numerical instability inherent for incorrectly posed formulations. Other possible numerical methods solving $(\mathbf{u}, \mathbf{p})$ problem in $2 \mathrm{D}$ are based on finite element or on boundary element techniques, which still necessitates the application of a regularisation technique to provide 
stability of the solution. It is understood that the use of standard regularisation techniques (as Tikhonov's or SVD regularisations) is capable to stabilize the solution (e.g. [4]-[7]), but for large systems their application is limited. Therefore, the development of alternative techniques for 3D case is necessary. Shwab [8] has shown that the Cauchy problem of 3D elasticity can be reduced to the Moisil and Theodoresco system [9], that provides generalisation of the integral Cauchy theorem from $2 \mathrm{D}$ into $3 \mathrm{D}$ and proved the uniqueness theorem for the solution of the problem. He also noticed that the problem is conditionally ill-posed as it can possess unstable solutions and investigated the accuracy of the solution by employing a boundary element technique. Galybin and Irša [10] have developed an FEM type technique for solving the Cauchy type problem for 3D harmonic functions complemented by the LSQR algorithm [11] for large systems. This allowed one to perform reconstruction of the contact stresses under a rigid circular punch applied to the boundary of elastic isotropic half-space by using the data of strain monitoring on the free surface of the half-space. This paper is aimed at the development of methods for solving the (u,p) problem for layered structures, in particular for the bi-material structure consisting of a coating bonded to a substrate. The main focus is to derive the integral relationships that can be useful for detection of delamination on the interface between the coating and the substrate. The work employs the method of spacious harmonic functions and the Fourier transform, which is widely used for solving direct boundary value problems for layered structures or bodies with planar cracks (e.g. [12], [13]).

\section{PROBLEM FORMULATION AND INTEGRAL EQUATIONS}

\subsection{General solution in terms of harmonic functions}

We consider an elastic layer $0<x_{3}<H$ with the moduli $G$ (shear modulus) and $\kappa$ (Muskhelishvili's constant) that can be fully or partly bonded to the half-space $x_{3}<0$ that has the moduli $G_{0}$ and $\kappa_{0}$ as shown in Fig. 1.

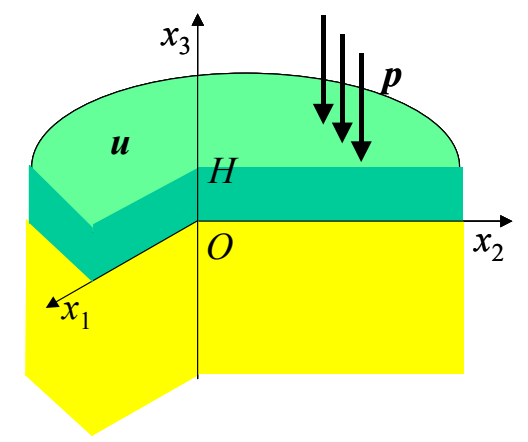

Figure 1: Geometry of the problem.

The boundary conditions are as follows:

$\mathrm{BC} 1$. The stress and displacement vectors are both known on the boundary $x_{3}=H$;

$\mathrm{BC} 2$. The stress and displacement vectors are both continuous across the interface $x_{3}=0$.

It is understood that this formulation is overdetermined if $\mathrm{BC} 2$ holds on the entire boundary $x_{3}=0$. However, if a delamination occurs on a part of the interface, BC2 can be used for the sake of its detection by solving the Cauchy initial value problem, IVP, for the 
layer with $\mathrm{BC} 1$. Therefore, we mainly focus on the Cauchy IVP and then discuss its application for the crack detection. In either case the aim is to construct a system of governing integral equations for the problem. Based on the formulas form Sih and Liebowitz [14] it has been shown [15], [16] that the following combination of the stress and displacement components can be expressed in terms of three spacious harmonic functions $\varphi=\varphi\left(x_{1}, x_{2}, x_{3}\right), \psi=\psi\left(x_{1}, x_{2}, x_{3}\right)$ and $\chi=\chi\left(x_{1}, x_{2}, x_{3}\right)$ as follows:

$$
\begin{gathered}
\partial_{1} u_{2}-\partial_{2} u 1=2(1-v) \chi, \\
\partial_{1} \sigma_{23}-\partial_{2} \sigma_{13}=2 G(1-v) \partial_{3} \chi, \\
\frac{\partial_{1} \sigma_{13}+\partial_{2} \sigma_{23}}{2 G}=-\partial_{33} \psi-x_{3} \partial_{333}(\varphi+\psi)=\left(\partial_{11}+\partial_{22}\right)\left(\psi+x_{3} \partial_{3}(\varphi+\psi)\right), \\
\partial_{1} u_{1}+\partial_{2} u_{2}=-(1-2 v) \partial_{3} \phi-2(1-v) \partial_{3} \psi-x_{3} \partial_{33}(\phi+\psi)= \\
=-\partial_{3}((1-2 v) \phi+2(1-v) \psi)+x_{3}\left(\partial_{11}+\partial_{22}\right)(\phi+\psi), \\
u_{3}=-2(1-v) \varphi-(1-2 v) \psi+x_{3} \partial_{3}(\varphi+\psi), \\
\frac{\sigma_{33}}{2 G}=-\partial_{3} \varphi+x_{3} \partial_{33}(\varphi+\psi) .
\end{gathered}
$$

Here, the symbols $\partial_{k}$ stand for the derivative with respect to the $k$-th variable and since the functions $\varphi, \psi$ and $\chi$ are harmonic we have used the following obvious relationship that is valid for any spacious harmonic function $\Phi=\Phi\left(x_{1}, x_{2}, x_{3}\right)$,

$$
\partial_{33} \Phi=-\partial_{11} \Phi-\partial_{22} \Phi \text {. }
$$

These equations are valid for the layer as well as for the half-space, but in the latter case the moduli $G$ and $\kappa$ should by replaced by $G_{0}$ and $\kappa_{0}$ respectively. As evident from the system it can be decomposed into two independent groups of equations. Eqns (1) and (2) form the first group for the determination of the function $\chi$ and four eqns (eqns (3)-(6)) form the second group for the determination of $\phi$ and $\psi$.

\subsection{Fourier transform of 3D harmonic functions}

We further use the double Fourier transform applied with respect to $x_{1}$ and $x_{2}$ in the form

$$
\begin{gathered}
\mathbf{D F}\left(Y\left(x_{1}, x_{2}\right)\right) \equiv \hat{Y}\left(s_{1}, s_{2}\right)=\int_{-\infty}^{\infty} \int_{-\infty}^{\infty} Y\left(x_{1}, x_{2}\right) e^{-i s_{1} x_{1}} e^{-i s_{2} x_{2}} d x_{1} d x_{2} \\
\mathbf{D F}^{-1}\left(\hat{Y}\left(s_{1}, s_{2}\right)\right) \equiv Y\left(x_{1}, x_{2}\right)=\frac{1}{(2 \pi)^{2}} \int_{-\infty}^{\infty} \int_{-\infty}^{\infty} \hat{Y}\left(s_{1}, s_{2}\right) e^{-i s_{1} x_{1}} e^{-i s_{2} x_{2}} d s_{1} d s_{2}
\end{gathered}
$$

By applying eqn (8) to eqn (7), one finds

$$
\partial_{33} \hat{\Phi}\left(s_{1}, s_{2}, x_{3}\right)-\lambda^{2}\left(s_{1}, s_{2}\right) \hat{\Phi}\left(s_{1}, s_{2}, x_{3}\right)=0, \quad \lambda\left(s_{1}, s_{2}\right)=\sqrt{s_{1}^{2}+s_{2}^{2}} .
$$


The solution for $\hat{\Phi}\left(s_{1}, s_{2}, x_{3}\right)$ and its normal derivative can be presented in the form

$$
\begin{gathered}
\hat{\Phi}\left(s_{1}, s_{2}, x_{3}\right)=\hat{\Phi}_{1}\left(s_{1}, s_{2}\right) e^{-\lambda\left(s_{1}, s_{2}\right) x_{3}}+\hat{\Phi}_{2}\left(s_{1}, s_{2}\right) e^{\lambda\left(s_{1}, s_{2}\right)\left(x_{3}-H\right)}, \\
\frac{\partial_{3} \hat{\Phi}\left(s_{1}, s_{2}, x_{3}\right)}{\lambda\left(s_{1}, s_{2}\right)}=-\hat{\Phi}_{1}\left(s_{1}, s_{2}\right) e^{-\lambda\left(s_{1}, s_{2}\right) x_{3}}+\hat{\Phi}_{2}\left(s_{1}, s_{2}\right) e^{\lambda\left(s_{1}, s_{2}\right)\left(x_{3}-H\right)},
\end{gathered}
$$

for the layer $0 \leq x_{3} \leq H$ and

$$
\begin{gathered}
\hat{\Phi}\left(s_{1}, s_{2}, x_{3}\right)=\hat{\Phi}_{0}\left(s_{1}, s_{2}\right) e^{\lambda\left(s_{1}, s_{2}\right) x_{3}}, \\
\partial_{3} \hat{\Phi}\left(s_{1}, s_{2}, x_{3}\right)=\hat{\Phi}_{0}\left(s_{1}, s_{2}\right) \lambda\left(s_{1}, s_{2}\right) e^{\lambda\left(s_{1}, s_{2}\right) x_{3}},
\end{gathered}
$$

for the half-space $x_{3} \leq 0$.

It is evident from eqns (12) and (13) that one cannot simultaneously impose two independent boundary conditions of the Dirichlet and Neumann type on the boundary of half-space as the problem becomes overspecified. This fact will be used further on for outlining a procedure for crack detection.

\subsection{Solution for the layer in Fourier space}

Let us consider the Cauchy IVP for the layer assuming that the boundary values of both the function and its normal derivatives are given on the boundary $x_{3}=H$. It follows from eqns (10) and (11) that for $0 \leq x_{3} \leq H$

$$
\begin{gathered}
\hat{\Phi}\left(s_{1}, s_{2}, x_{3}\right)+\frac{\partial_{3} \hat{\Phi}\left(s_{1}, s_{2}, x_{3}\right)}{\lambda\left(s_{1}, s_{2}\right)}=2 \hat{\Phi}_{2}\left(s_{1}, s_{2}\right) e^{\lambda\left(s_{1}, s_{2}\right)\left(x_{3}-H\right)}, \\
\hat{\Phi}\left(s_{1}, s_{2}, x_{3}\right)-\frac{\partial_{3} \hat{\Phi}\left(s_{1}, s_{2}, x_{3}\right)}{\lambda\left(s_{1}, s_{2}\right)}=2 \hat{\Phi}_{1}\left(s_{1}, s_{2}\right) e^{-\lambda\left(s_{1}, s_{2}\right) x_{3}} .
\end{gathered}
$$

On the boundary $x_{3}=H$

$$
\begin{gathered}
\hat{\Phi}\left(s_{1}, s_{2}, H\right)+\frac{\partial_{3} \hat{\Phi}\left(s_{1}, s_{2}, H\right)}{\lambda\left(s_{1}, s_{2}\right)}=2 \hat{\Phi}_{2}\left(s_{1}, s_{2}\right), \\
\hat{\Phi}\left(s_{1}, s_{2}, H\right)-\frac{\partial_{3} \hat{\Phi}\left(s_{1}, s_{2}, H\right)}{\lambda\left(s_{1}, s_{2}\right)}=2 \hat{\Phi}_{1}\left(s_{1}, s_{2}\right) e^{-\lambda\left(s_{1}, s_{2}\right) H} .
\end{gathered}
$$

On the interface $x_{3}=0$

$$
\begin{gathered}
\hat{\Phi}\left(s_{1}, s_{2}, 0\right)+\frac{\partial_{3} \hat{\Phi}\left(s_{1}, s_{2}, 0\right)}{\lambda\left(s_{1}, s_{2}\right)}=2 \hat{\Phi}_{2}\left(s_{1}, s_{2}\right) e^{-\lambda\left(s_{1}, s_{2}\right) H}, \\
\hat{\Phi}\left(s_{1}, s_{2}, 0\right)-\frac{\partial_{3} \hat{\Phi}\left(s_{1}, s_{2}, 0\right)}{\lambda\left(s_{1}, s_{2}\right)}=2 \hat{\Phi}_{1}\left(s_{1}, s_{2}\right) .
\end{gathered}
$$


It is evident from eqns (16) to (19) that the initial value problem for the harmonic function is readily found by known values of the function and its normal derivative at either end $\left(x_{3}=0\right.$ or $\left.x_{3}=H\right)$. Thus, if the initial conditions are specified for $x_{3}=H$, then

$$
\begin{gathered}
2 \hat{\Phi}\left(s_{1}, s_{2}, x_{3}\right)=\left(\hat{\Phi}\left(s_{1}, s_{2}, H\right)-\frac{\partial_{3} \hat{\Phi}\left(s_{1}, s_{2}, H\right)}{\lambda\left(s_{1}, s_{2}\right)}\right) e^{-\lambda\left(s_{1}, s_{2}\right)\left(x_{3}-H\right)}+ \\
+\left(\hat{\Phi}\left(s_{1}, s_{2}, H\right)+\frac{\partial_{3} \hat{\Phi}\left(s_{1}, s_{2}, H\right)}{\lambda\left(s_{1}, s_{2}\right)}\right) e^{\lambda\left(s_{1}, s_{2}\right)\left(x_{3}-H\right)}, 0 \leq x_{3} \leq H .
\end{gathered}
$$

It is evident, however, that the application of the inverse Fourier transform cannot be performed directly due to positive power of the first exponent in the right hand of eqn (20). This fact also indicates that the solution of the initial value problem for harmonic functions is unstable.

Let us find the relationships between the Fourier transforms on the boundaries. It follows from eqns (16) and (18) that

$$
\hat{\Phi}\left(s_{1}, s_{2}, 0\right)+\frac{\partial_{3} \hat{\Phi}\left(s_{1}, s_{2}, 0\right)}{\lambda\left(s_{1}, s_{2}\right)}=\left(\hat{\Phi}\left(s_{1}, s_{2}, H\right)+\frac{\partial_{3} \hat{\Phi}\left(s_{1}, s_{2}, H\right)}{\lambda\left(s_{1}, s_{2}\right)}\right) e^{-\lambda\left(s_{1}, s_{2}\right) H} .
$$

From eqns (17) and (19), one finds

$$
\hat{\Phi}\left(s_{1}, s_{2}, H\right)-\frac{\partial_{3} \hat{\Phi}\left(s_{1}, s_{2}, H\right)}{\lambda\left(s_{1}, s_{2}\right)}=\left(\hat{\Phi}\left(s_{1}, s_{2}, 0\right)-\frac{\partial_{3} \hat{\Phi}\left(s_{1}, s_{2}, 0\right)}{\lambda\left(s_{1}, s_{2}\right)}\right) e^{-\lambda\left(s_{1}, s_{2}\right) H} .
$$

These relationships are used further on for derivation of a system of integral equations.

\subsection{Reduction to integral equations}

Let us apply the inverse Fourier transform to eqns (21) and (22) and use the convolution theorem, which leads to the following system of integral equations of the Fredholm type of the first kind:

$$
\begin{gathered}
\Phi\left(x_{1}, x_{2}, 0\right)+\frac{1}{2 \pi} \int_{-\infty}^{\infty} \int_{-\infty}^{\infty} \frac{\partial_{3} \Phi(\xi, \eta, 0)}{\rho\left(\xi-x_{1}, \eta-x_{2}, 0\right)} d \xi d \eta= \\
=\frac{1}{2 \pi} \int_{-\infty}^{\infty} \int_{-\infty}^{\infty}\left(\frac{H \Phi(\xi, \eta, H)}{\rho^{3}\left(\xi-x_{1}, \eta-x_{2}, H\right)}+\frac{\partial_{3} \Phi(\xi, \eta, H)}{\rho\left(\xi-x_{1}, \eta-x_{2}, H\right)}\right) d \xi d \eta, \quad-\infty<x_{1}, x_{2}<\infty \\
\quad \frac{1}{2 \pi} \int_{-\infty}^{\infty} \int_{-\infty}^{\infty}\left(\frac{H \Phi(\xi, \eta, 0)}{\rho^{3}\left(\xi-x_{1}, \eta-x_{2}, H\right)}-\frac{\partial_{3} \Phi(\xi, \eta, 0)}{\rho\left(\xi-x_{1}, \eta-x_{2}, H\right)}\right) d \xi d \eta= \\
=\Phi\left(x_{1}, x_{2}, H\right)-\frac{1}{2 \pi} \int_{-\infty}^{\infty} \int_{-\infty}^{\infty} \frac{\partial_{3} \Phi(\xi, \eta, H)}{\rho\left(\xi-x_{1}, \eta-x_{2}, 0\right)} d \xi d \eta, \quad-\infty<x_{1}, x_{2}<\infty .
\end{gathered}
$$


Here,

$$
\rho\left(x_{1}, x_{2}, x_{3}\right)=\sqrt{x_{1}^{2}+x_{2}^{2}+x_{3}^{2}},
$$

whose reciprocal $(1 / \rho)$ is a harmonic function and the following integrals [13] have been used taking into account that $\rho$ is even with respect to all variables:

$$
\begin{aligned}
& \frac{2}{\pi} \int_{0}^{\infty} \int_{0}^{\infty} \frac{1}{\lambda(\alpha, \beta)} e^{-\lambda(\alpha, \beta) z} \cos (\alpha x) \cos (\beta y) d \alpha d \beta=\frac{1}{\rho(x, y, z)}, \\
& \frac{2}{\pi} \int_{0}^{\infty} \int_{0}^{\infty} e^{-\lambda(\alpha, \beta) z} \cos (\alpha x) \cos (\beta y) d \alpha d \beta=\frac{z}{\rho^{3}(x, y, z)}, \quad z>0 .
\end{aligned}
$$

Note that the integral in eqn (27) can be obtained by differentiation of eqn (26) with respect to $x_{3}$.

The system eqns (23) and (24) is sought system of governing integral equations for the elastic layer.

\section{THE CASE OF NORMAL LOADING}

\subsection{Solution for the layer}

Let us consider a special case of normal loading of the upper boundary $\left(x_{3}=H>0\right)$ of the layer, which is most interesting from practical point of view. Assume $\sigma_{13}\left(x_{1}, x_{2}, x_{3}\right)=0$ and $\sigma_{23}\left(x_{1}, x_{2}, x_{3}\right)=0$ in the system eqns (1)-(6) and introduce the following notations for known (measured) combinations of the displacements on $x_{3}=H$ :

$$
\begin{aligned}
& 2(1-v) U\left(x_{1}, x_{2}\right)=u_{3}\left(x_{1}, x_{2}, H\right), \\
& 2(1-v) R\left(x_{1}, x_{2}\right)=\partial_{1} u_{2}\left(x_{1}, x_{2}, H\right)-\partial_{2} u_{1}\left(x_{1}, x_{2}, H\right), \\
& 2(1-v) S\left(x_{1}, x_{2}\right)=\partial_{1} u_{1}\left(x_{1}, x_{2}, H\right)+\partial_{2} u_{2}\left(x_{1}, x_{2}, H\right) .
\end{aligned}
$$

The first group of eqns (1) and (2) takes the form

$$
\chi\left(x_{1}, x_{2}, H\right)=R\left(x_{1}, x_{2}\right), \quad \partial_{3} \chi\left(x_{1}, x_{2}, H\right)=0 .
$$

The second group is obtained by summing eqns (4) and (6) and by taking into account that the last two terms in eqn (5) can be neglected due to homogeneity of eqn (3) in the case of normal load. Therefore, we arrive at the problem of finding the sum of two unknown harmonic functions as follows:

$$
\begin{gathered}
u_{3}=-2(1-v)(\varphi+\psi), \\
\partial_{1} u_{1}+\partial_{2} u_{2}+\frac{\sigma_{33}}{2 G}=-2(1-v) \partial_{3}(\varphi+\psi) .
\end{gathered}
$$


As soon as the sum $\varphi+\psi$ is determined one can derive the solutions for $\varphi$ and $\psi$ and their normal derivatives by using eqns (3)-(5). By introducing the auxiliary harmonic function $\omega=-\varphi-\psi$ and employing eqn (28), one obtains the following initial value problem:

$$
\omega\left(x_{1}, x_{2}, H\right)=U\left(x_{1}, x_{2}\right), \quad \partial_{3} \omega\left(x_{1}, x_{2}, H\right)=S\left(x_{1}, x_{2}\right)+P\left(x_{1}, x_{2}\right),
$$

where the function $P\left(x_{1}, x_{2}\right)=\sigma_{33}\left(x_{1}, x_{2}, H\right)(2 D)^{-1}$ is known exactly in contrast to the other terms on the right-hand sides of eqns (29) and (32) that contain measurement errors.

It is physically more realistic to measure displacements (strains) on the stress-free surfaces. In this case the dimensions of the zones of load application should be relatively small compared with the areas of displacement monitoring. This is valid in case of concentrated loads, which ensures that displacements are known over almost the whole boundary except for a finite number of points and thus all $\mathrm{BC} 1$ are satisfied. For simplicity it is assumed that all the loads acting on the surface $x_{3}=H$ are concentrated forces applied at different locations. For a normal concentrated load of intensity $P_{0}$ applied at the origin the function $P\left(x_{1}, x_{2}\right)=P_{0} \delta\left(x_{1}, x_{2}\right)$, where $\delta\left(x_{1}, x_{2}\right)$ is the Delta function. Its Fourier transform is constant $P_{0}$. In order to keep the layer in equilibrium the resultant normal stress acting on the opposite side of the layer should have the same value $P_{0}$ regardless of the its distribution over the plane $x_{3}=0$. If the load on the opposite side is axisymmetric then all the conditions of global equilibrium are satisfied. This load can be selected, for example, as $P_{0} \exp \left(-x_{1}^{2}-x_{2}^{2}\right) / \pi$, which emphasizes the fact that the stress and displacement vectors in the layer can possess polar symmetry. Let $\omega_{0}$ be a harmonic function that presents the solution of the boundary value problem in normal stresses for the elastic layer. Then we can seek for a solution of the initial value problem with modified conditions eqn (32) as follows:

$$
\omega\left(x_{1}, x_{2}, H\right)=U\left(x_{1}, x_{2}\right)-U_{0}\left(x_{1}, x_{2}\right), \quad \partial_{3} \omega\left(x_{1}, x_{2}, H\right)=S\left(x_{1}, x_{2}\right)-S_{0}\left(x_{1}, x_{2}\right),(33)
$$

where $U_{0}$ and $S_{0}$ are the combinations of the displacement in eqn (28) that correspond to the solution of the BVP in terms of stresses. In the case of single concentrated load these functions possess polar symmetry, i.e. they depend only on the polar radius. In the case of several concentrated forces applied on $x_{3}=H$ one should use superposition of the particular solutions for every force and thus the polar symmetry does not take place anymore. We do not use symmetry further.

\subsubsection{System for $\chi$}

In this case, we have to use eqn (29). Then the system eqns (23) and (24) becomes $\left(-\infty<x_{1}, x_{2}<\infty\right)$ :

$$
\left\{\begin{array}{c}
\chi\left(x_{1}, x_{2}, 0\right)+\frac{1}{2 \pi} \int_{-\infty}^{\infty} \int_{-\infty}^{\infty} \frac{\partial_{3} \chi(\xi, \eta, 0)}{\rho\left(\xi-x_{1}, \eta-x_{2}, 0\right)} d \xi d \eta=\frac{H}{2 \pi} \int_{-\infty-\infty}^{\infty} \int_{-\infty}^{\infty} \frac{R(\xi, \eta)}{\rho^{3}\left(\xi-x_{1}, \eta-x_{2}, H\right)} d \xi d \eta \\
\frac{1}{2 \pi} \int_{-\infty-\infty}^{\infty} \int_{-\infty}^{\infty}\left(\frac{H \chi(\xi, \eta, 0)}{\rho^{3}\left(\xi-x_{1}, \eta-x_{2}, H\right)}-\frac{\partial_{3} \chi(\xi, \eta, 0)}{\rho\left(\xi-x_{1}, \eta-x_{2}, H\right)}\right) d \xi d \eta=R\left(x_{1}, x_{2}\right) .
\end{array}\right.
$$

It follows from eqns (21) and (22) with the use of eqn (29) that this system is consistent only when the following relationship between $\chi\left(x_{1}, x_{2}, 0\right)$ and $\partial_{3} \chi\left(x_{1}, x_{2}, 0\right)$ holds: 


$$
\begin{gathered}
\chi\left(x_{1}, x_{2}, 0\right)+\frac{1}{2 \pi} \int_{-\infty}^{\infty} \int_{-\infty}^{\infty} \frac{\partial_{3} \chi(\xi, \eta, 0)}{\rho\left(\xi-x_{1}, \eta-x_{2}, 0\right)} d \xi d \eta= \\
=\frac{1}{2 \pi} \int_{-\infty}^{\infty} \int_{-\infty}^{\infty}\left(\frac{2 H \chi(\xi, \eta, 0)}{\rho^{3}\left(\xi-x_{1}, \eta-x_{2}, 2 H\right)}-\frac{\partial_{3} \chi(\xi, \eta, 0)}{\rho\left(\xi-x_{1}, \eta-x_{2}, 2 H\right)}\right) d \xi d \eta .
\end{gathered}
$$

\subsubsection{System for $\omega$}

Substitution of eqn (33) into eqns (23)-(24) results in $\left(-\infty<x_{1}, x_{2}<\infty\right)$ :

$$
\left\{\begin{array}{c}
\omega\left(x_{1}, x_{2}, 0\right)+\frac{1}{2 \pi} \int_{-\infty-\infty}^{\infty} \int_{-\infty}^{\infty} \frac{\partial_{3} \omega(\xi, \eta, 0)}{\rho\left(\xi-x_{1}, \eta-x_{2}, 0\right)} d \xi d \eta=F_{1}\left(x_{1}, x_{2}, H\right) \\
\frac{1}{2 \pi} \int_{-\infty-\infty}^{\infty} \int_{-\infty}^{\infty}\left(\frac{H \omega(\xi, \eta, 0)}{\rho^{3}\left(\xi-x_{1}, \eta-x_{2}, H\right)}-\frac{\partial_{3} \omega(\xi, \eta, 0)}{\rho\left(\xi-x_{1}, \eta-x_{2}, H\right)}\right) d \xi d \eta=F_{2}\left(x_{1}, x_{2}\right)
\end{array}\right.
$$

Here the right-hand side of the system is as follows:

$$
\begin{gathered}
F_{1}\left(x_{1}, x_{2}, H\right)=\frac{1}{2 \pi} \int_{-\infty}^{\infty} \int_{-\infty}^{\infty}\left(\frac{H\left(U(\xi, \eta)-U_{0}(\xi, \eta)\right)}{\rho^{3}\left(\xi-x_{1}, \eta-x_{2}, H\right)}+\frac{S(\xi, \eta)-S_{0}(\xi, \eta)}{\rho\left(\xi-x_{1}, \eta-x_{2}, H\right)}\right) d \xi d \eta \\
F_{2}\left(x_{1}, x_{2}\right)=U\left(x_{1}, x_{2}\right)-U_{0}\left(x_{1}, x_{2}\right)-\frac{1}{2 \pi} \int_{-\infty}^{\infty} \int_{-\infty}^{\infty} \frac{S(\xi, \eta)-S_{0}(\xi, \eta)}{\rho\left(\xi-x_{1}, \eta-x_{2}, 0\right)} d \xi d \eta
\end{gathered}
$$

As soon as the solution of eqn (36) is found one can find the total solution for the case of concentrated loads by summing $\omega$ with $\omega_{0}$. As a result, we determine the combination $\partial_{1} u_{1}+\partial_{2} u_{2}+\sigma_{33}(2 G)^{-1}$ and the normal displacement $u_{3}$. The normal stresses on the interface $x_{3}=0$ is found by differentiating eqn (5) with respect to $x_{3}$ and summing with eqn (6), which results in

$$
\frac{\sigma_{33}\left(x_{1}, x_{2}, 0\right)}{2 G}=(1-2 v) \partial_{3} \omega\left(x_{1}, x_{2}, 0\right)+\partial_{3} u_{3}\left(x_{1}, x_{2}, 0\right) .
$$

\subsection{Numerical approach}

Several standard methods can be employed for solving the system of integral eqn (36) taking into account that the second integral equation has regular kernels while the first one has a weak singularity and therefore the second term in its left hand side should be treated as a convergent improper integral.

Therefore, eqn (34) can be referred to as a system of Fredholm integral equations of the first kind, which usually possess unstable solutions. Alternatively, one can use the Trefftz-type approach that demonstrated satisfactory performance for solving unstable Fredholm equations when complemented by the SVD regularisation (see Galybin [15] for detail). 
Let us seek the function $\omega$ and its derivative with respect to $x_{3}$ (that is also harmonic) as two linear combinations of the radial basis functions $1 / \rho$ with the nodes $\left(\xi_{k}, \eta_{k}, \zeta_{k}\right)$ lying outside the layer

$$
\begin{gathered}
\omega\left(x_{1}, x_{2}, x_{3}\right)=\sum_{k=1}^{N} \frac{a_{k}}{\rho\left(x_{1}-\xi_{k}, x_{2}-\eta_{k}, x_{3}-\xi_{k}\right)}, \\
\partial_{3} \omega\left(x_{1}, x_{2}, x_{3}\right)=\sum_{k=1}^{N} \frac{b_{k}}{\rho\left(x_{1}-\xi_{k}, x_{2}-\eta_{k}, x_{3}-\xi_{k}\right)} .
\end{gathered}
$$

Then the following system of ( $3 M$ by $2 N$ ) linear algebraic equations can be formed for determination of unknown coefficient $a_{k}$ and $b_{k}$ :

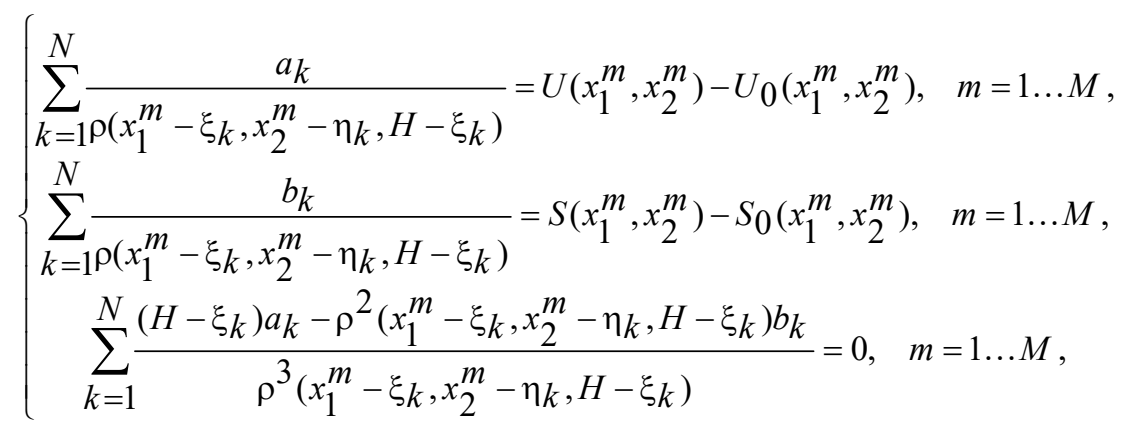

where $x_{1}^{m}, x_{2}^{m}$ are collocation points $m=1 \ldots M$.

This system is overdetermined if $3 M>2 N$ and therefore its approximate solution can be found by the least square method. It should be mentioned that a regularisation procedure is necessary in the case when the condition number of the matrix is large. As shown in Galybin [15] the SVD regularisation is efficient for medium size matrices while for large matrices one can use Tikhonov's regularisation.

\subsection{A procedure for interface delamination detection}

The solution for the layer can be used to suggest a procedure for checking if the layer and the half-space are fully bonded or delamination has occurred on the interface $x_{3}=0$. In the case of full bonding between the layer and the half-space the continuity of the stress and displacement vectors on the interface $x_{3}=0$ should be satisfied. Otherwise they are violated. As follows from the solution for the lower half-space:

$$
\partial_{3} \hat{\Phi}\left(s_{1}, s_{2}, 0\right)=\lambda\left(s_{1}, s_{2}\right) \hat{\Phi}\left(s_{1}, s_{2}, 0\right),
$$

Therefore,

$$
\lambda\left(s_{1}, s_{2}\right) \hat{u}_{3}\left(s_{1}, s_{2}, 0\right)=\partial_{1} \hat{u}_{2}\left(s_{1}, s_{2}, 0\right)+\partial_{1} \hat{u}_{2}\left(s_{1}, s_{2}, 0\right)+\frac{1}{2 G_{0}} \hat{\sigma}_{33}\left(s_{1}, s_{2}, 0\right) .
$$

After application of the inverse Fourier transform to eqn (44), it can be presented in one of the following equivalent forms (we assume that the solutions are smooth enough): 


$$
u_{3}\left(x_{1}, x_{2}, 0\right)=\frac{1}{2 \pi} \int_{-\infty}^{\infty} \int_{-\infty}^{\infty} \frac{\partial_{1} u_{2}(\xi, \eta, 0)+\partial_{1} u_{2}(\xi, \eta, 0)+\frac{1}{2 G_{0}} \sigma_{33}(\xi, \eta, 0)}{\rho\left(\xi-x_{1}, \eta-x_{2}, 0\right)} d \xi d \eta
$$

or

$$
\begin{gathered}
\frac{-1}{2 \pi} \int_{-\infty}^{\infty} \int_{-\infty}^{\infty} \frac{\left(\partial_{11}+\partial_{22}\right) u_{3}(\xi, \eta, 0)}{\rho\left(\xi-x_{1}, \eta-x_{2}, 0\right)} d \xi d \eta= \\
=\partial_{1} u_{2}\left(x_{1}, x_{2}, 0\right)+\partial_{1} u_{2}\left(x_{1}, x_{2}, 0\right)+\frac{1}{2 G_{0}} \sigma_{33}\left(x_{1}, x_{2}, 0\right) .
\end{gathered}
$$

The solution of the Cauchy IVP for the layer determines two harmonic functions, one for the combination $\partial_{1} u_{1}+\partial_{2} u_{2}+\sigma_{33}(2 G)^{-1}$ and another for the normal displacement $u_{3}$ in the layer as well as their boundary values. The normal stress is also known from eqn (39). Therefore, one can substitute these solutions into either eqn (45) or eqn (46) to check if the initial conditions given by eqn (28) satisfy them. Given that the strain/displacement measurements on the boundary $x_{3}=H$ are subjected to errors, eqns (45) and (46) should be satisfied approximately. Discussion of the choice of a proper level of approximation is beyond the scope of this paper. We should assume that if both eqns (45) and (46) are not fulfilled, then we expect the presence of a delamination on the interface. It can also be located by calculating the normal stresses that have to be close to zero on the surface of delamination.

\section{CONCLUSIONS}

This study concerns the 3D elastic problem for the coating bonded to a substrate from a dissimilar elastic material. We presented the system of integral equations for the Cauchy initial value problem for an elastic layer that followed from the analytical solution in the Fourier space. It is shown that the solution of the system is unstable because the system consists of the Fredholm equations of the first kind. A numerical procedure is suggested that can hamper instability. We have also presented the relationships that have to be imposed on the initial conditions that satisfy continuity of the stress and displacement vectors on the interface between the layer and the half-space.

\section{ACKNOWLEDGEMENT}

This work was supported by the grant of the Government of the Russian Federation No. 14.Z50.31.0046.

\section{REFERENCES}

[1] Galybin, A.N. \& Rogerson, G.A., Ill-posed problem of the Cauchy type for an elastic strip. Mathematics and Mechanics of Solids, 2019. (E-pub ahead of print). DOI: $10.1177 / 1081286519826395$.

[2] Shvab, A.A., Incorrectly posed static problems of elasticity. Solid Mechanics, 24, pp. 98-106, 1989.

[3] Bonnet, M. \& Constantinescu, A., Inverse problems in elasticity. Inverse Problems, 21, pp. R1-R50, 2005. 
[4] Marin, L., Elliott, L., Ingham, D.B. \& Lesnic, D., Boundary element method for the Cauchy problem in linear elasticity. Engineering Analysis with Boundary Elements, 25(9), pp. 783-793, 2001.

[5] Marin, L. \& Lesnic, D., Boundary element solution for the Cauchy problem in linear elasticity using singular value decomposition. Computer Methods in Applied Mechanics and Engineering, 191(6), pp. 3257-3270, 2002.

[6] Marin, L. \& Lesnic, D., The method of fundamental solutions for inverse boundary value problems associated with the two-dimensional biharmonic equation. Mathematical and Computer Modelling, 42(3-4), pp. 261-278, 2005.

[7] Galybin, A.N., A method for determination of stress distributions in the process zone ahead of a 2D crack. "Moving Boundaries VI" Computational Modelling of Free and Moving Boundary Problems, eds B. Sarler \& C.A. Brebbia, WIT Press: Southampton, pp. 243-252, 2001.

[8] Schwab, A.A., The inverse problem of elasticity theory: Application of the boundary integral equation for the holomorphic vector. Physics of the Solid Earth, 30(4), pp. 342-348, 1994.

[9] Moisil, G.C. \& Theodoresco, N., Fonctions holomorphes dans l'espace. Mathematica, 5, pp. 141-153, 1931.

[10] Galybin, A.N. \& Irša, J., On reconstruction of 3D harmonic functions from discrete data. Proceedings of Royal Society A, 466(2119), pp. 1935-1955, 2010.

[11] Paige, C.C. \& Saunders, M.A., LSQR: An algorithm for sparse linear equations and sparse least squares. ACM Transactions on Mathematical Software, 8, pp. 43-71, 1982.

[12] Shevljakov, Y.A., Matrix Algorithms in the Theory of Elasticity of Inhomogeneous Media, Vischa Schola: Kiev and Odessa, p. 216, 1977. (In Russian.)

[13] Vafa, J.P. \& Fariborz, S.J., Analysis of rectangular cracks in elastic bodies. Theoretical and Applied Fracture Mechanics, 87, pp. 78-90, 2017.

[14] Sih, G.C. \& Liebowitz, H., Mathematical theories on brittle fracture. Fracture: An Advanced Treatise, vol. 2, ed. H. Liebowitz, Academic Press: New York, 1968.

[15] Galybin, A.N., Contact inverse problem for an elastic half-space. Engineering Analysis with Boundary Elements, 68, pp. 35-41, 2016.

[16] Galybin, A.N., Integral equations and Gauss-Chebyshev quadratures for planar rectangular cracks. International Journal of Mechanical Sciences, 146-147, pp. 272$279,2018$. 